KN Kartographische Nachrichten Journal of Cartography and Geographic Information 67. Jahrgang, Februar 2017, Heft 1 Herausgeber: Deutsche Gesellschaft für Kartographie e.V. (DGfK) - Gesellschaft für Kartographie und Geomatik, vertreten durch den Präsidenten Prof. Dr. Manfred Weisensee

Organ der Deutschen Gesellschaft für Kartographie e.V. (DGfK), der Schweizerischen Gesellschaft für Kartografie (SGK) und der Österreichischen Kartographischen Kommission (ÖKK) in der Österreichischen Gesellschaft für Geographie (ÖGG).

Schriftleitung

Hauptschriftleiter

Prof. Dr. rer. nat. habil. Mark Vetter (zuständig für Aufsätze, Berichte). Hochschule Karlsruhe - Technik und Wirtschaft, Moltkestr 30, 76133 Karlsruhe; redaktion@kartographischenachrichten.de, Fon: 0049(0) 7219252599

Weitere Schriftleiter

Prof. Dr. phil. Frank Dickmann (Zweiter Schriftleiter, zuständig für Nachrichten aus Hochschulen und Institutionen, Rezensionen).

Ruhr-Universität Bochum, Geographisches Institut,

Postfach, 44780 Bochum; frank.dickmann@rub.de

Fon: 0049(0) 234 32-23379; Fax: 0049(0) 234 32-14964

Dipl.-Ing. Andreas Gollenstede (zuständig für Geoinformation

aktuell) Haareneschstr. 91, 26121 Oldenburg; kn@gollenstede.com Fon: 0049(0) 441 7779-545; Fax: 0049(0) 441 7779-750

Dr.-Ing. Thomas Chudy (zuständig für DGfK-, SKG- und ÖGG-

Nachrichten). Martin-Luther-Universität Halle-Wittenberg, Institut

für Agrar- und Ernährungswissenschaften, Karl-Freiherr-von-

fur Agrar- und Ernährungswissenschaften, Karr-Freiherr-von-
Fritsch-Straße 4,06120 Halle/Saale, Fon: 0049(0) 3455522448

Fritsch-Straße 4, $06120 \mathrm{Ha}$

Prof. Dr. Reinhard Zölitz (zuständig für Nachrichten aus Hochschulen und Institutionen), Institut für Geographie und Geologie, Kartographie und GIS, Friedrich-Ludwig-Jahn-Str. 16,

D-17489 Greifswald, Fon 03834864523, Fax:03834864501,

E-17489 Greitswald, Foelitz@uni-greifswald.de

Mitarbeit für die Schweiz: Prof. Dr. Lorenz Hurni. Institut für Kartografie, ETH Hönggerberg, 8093 Zürich; hurni@karto.baug.ethz ch. Fon: 004116333033 ; Fax: 00411631153

Mitarbeit für Österreich: Univ.-Prof. Dr. Wolfgang Kainz Institut für Geographie und Regionalforschung, Universität Wien, Universitätsstraße 7/5, 1010 Wien; wolfgang.kainz@univie.ac.at Fon: 0043 14277-8640; Fax: 0043 14277-9531

Ehrenschriftleiter

Prof, i. R. Dr. Jürgen Dodt Witten

Dr. rer. nat. h. c. Rolf Harbeck, Bonn

Editorial Board

Prof. Dr. H. Asche, Potsdam; Prof. Dr. Manfred Buchroithner, Dresden; Prof. Dr. Dirk Burghardt, Dresden; Prof. i. R. Dr. Jürgen Dodt, Witten:Prof. Dr. D. Dransch, Potsdam: Prof. Dr. M. Ehlers Osnabrück, Berlin; Prof. Dr. S. I. Fabrikant, Zürich, Schweiz; Ass.-Prof. Dr. S. Fuhrmann, Fairfax, USA: Prof. Dr. G. Gartner, Wien, Öss.-Profech;. Pr. Funr. Dr. D. Grünreich, Ronnenberg; Dr. Rolf Harbeck, Osterreich; Prof. Dr. D. Grünreich, Ronnenberg; Dr. Rolf Haroeck Bonn; Prof. Dr. A. Hüttermann, Ludwigsburg; Prof. Dr. L. Hurni, Zürich; Prof. Dr. W. Kainz, Wien; Prof. Dr. P. Kammerer, München Prof. Dr. W. G. Koch, Dresden; Prof. Dr. Menno-Jan Kraak, Twente, Netherlands; Prof. Dr. Jukka Krisp, Augsburg; Prof. Dr.-Ing. L. Meng, München; Prof. Dr. J. Schiewe, Hamburg: PD Dr.-Ing J. Schoppmever, Bonn: Prof. Dr. J. Schweikart, Berlin Prof. Dr.-Ing. M. Sester, Hannover; Prof. Dr. J. Siemer, Regina, Kanada; Prof. Dr. R. Zölitz, Greifswald.

Manuskripte richten Sie bitte an den zuständigen Schriftleiter. Hinweise zur Gestaltung des Manuskripts finden Sie unter www.kartographische-nachrichten.de > Autorenhinweise. Reviewverfahren

Wissenschaftliche Beiträge werden im Rahmen eines Peer-ReviewVerfahrens begutachtet. Trotz sorgfältiger Redigierung übernehmen Herausgeber, Redaktion und Verlag keine Haftung für die inhaltlich Richtigkeit der Veröffentlichungen.

Die KN Kartographische Nachrichten ist in der internationalen Zitationsdatenbank Scopus gelistet

Die DGfK ist Mitglied der

Internationalen Kartographischen

Vereinigung IKV/ICA.

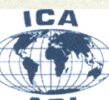

Verlag: Kirschbaum Verlag $\mathrm{GmbH}$, SiegfriedstraBe 28 D-53179 Bonn / Postfach 210209, D-53157 Bonn,

Fon: (0228) 95453-0, Fax: (0228) 95453-27

Internet: http://www.kirschbaum.de, E-Mail: info@kirschbaum.de

Anzeigenleiter: Volker Rutkowski; zurzeit ist Anzeigenpreisliste

Nr. 52 vom 1.10.2016 gültig

Bezugspreise und ISSN: Inland/Ausland Jahresabonnement

inkl. E-Paper und elektronischem Archiv 74,- Euro zzgl.

Versand 9,80,- Euro (jeweils einschl. MwSt.).

Einzelheft 18,- Euro zzgl. Versand

Die Zeitschrift erscheint alle zwei Monate. ISSN 0022-9164.

Kündigungsfrist 6 Wochen zum Auslaufen des Abonnement-

Zeitraumes.

Satz: EMS Eckert Medienservice, 53359 Rheinbach

Druck: johnen-druck GmbH \& Co. KG, Industriegebiet Bornwiese,

54470 Bernkastel-Kues

\title{
Ein Plädoyer für mehr Berichte aus der Praxis
}

\section{Liebe Kolleginnen und Kollegen,}

ich hoffe, Sie sind gut im neuen Jahr angekommen!

Immer wieder höre ich die Bitte: „Herr Vetter, wir würden gerne mehr Berichte aus der Praxis lesen.“ Ich kann dies gut nachvollziehen. Ein Erfahrungsaustausch von Menschen aus der kartographischen Praxis ist $u$. a. der Mehrwert den die DGfK bietet für die vielen Kolleginnen und Kollegen, die in den privaten Büros, Verlagen, Ämtern, Hochschulen oder Forschungseinrichtungen täglich dem kartographischen Alltag nachgehen. Die jungen Geomatikerinnen und Geomatiker sind uns mit ihrer Mitgliedschaft nach der Ausbildung treu geblieben, um eine Weiterbildung über den Verein und

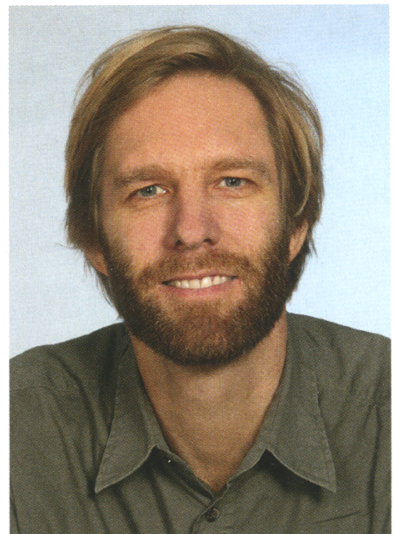

Mark Vetter über unsere Zeitschrift, als eine Möglichkeit, zu bekommen. Absolut richtig.

Sie können sich sicher sein, ich würde sehr gerne mehr Beiträge aus der Anwendung drucken. Es geht mir nicht alleine darum, in den KN nur Artikel auf Englisch zu modernsten wissenschaftlichen Erkenntnissen zu veröffentlichen. Uns - und damit meine ich die Redaktion und den ganzen Vorstand der DGfK - ist klar, dies alleine wäre nicht der richtige Weg. Daher möchte ich Sie herzlich darum bitten, schreiben Sie uns etwas. Lassen Sie uns teilhaben an Ihren Überlegungen und Erkenntnissen zu den Themen, die uns gegenwärtig fordern: Wie erleben Sie die aktuellen Veränderungen im Zuge der Digitalisierung in der Kartographie/Geomatik in Ihrem Arbeitsumfeld? Wie kommt die Umsetzung/Anwendung von europäischen oder nationalen Richtlinien zu Veränderungen in der Geoinformationswelt in Ihrer täglichen Praxis an? Können Sie heute noch so als Kartographin/Kartograph arbeiten, wie Sie sich dies vorgestellt haben, als Sie den Beruf ergriffen haben?

Diese Fragen bewegen unsere Gesellschaft. Wir freuen uns sehr, wenn Sie uns dazu Ihre Meinungsäußerungen senden könnten. Und bitte haben Sie keine Hemmungen. Die Kartographischen Nachrichten sind eine Vereinszeitschrift. Von Mitgliedern für Mitglieder. Und dies wird auch längerfristig so bleiben, allen anderen Gerüchten zum Trotz.

Im vorliegenden Heft lesen Sie wieder spannende Artikel aus aktuellen Projekten: Gerhard Fasching hat sich intensiv in seinem Beitrag mit einer Konzeption von Kartenund Geoinformationswerken für eine gemeinsame Außen- und Sicherheitspolitik der EU beschäftigt. Tilo Felgenhauer zeigt uns in seinem Beitrag die Sichtweise der Sozialgeographie auf das Thema VGI (Volunteered Geographic Information). Ferner lesen Sie einen Beitrag von dem Team um Frau Liqui Meng, welches sich seit Längerem mit den Herausforderungen der digitalen Kartographie beschäftigt. Dazu gehört zweifelsohne das Verständnis komplexer Eingabedaten, die Verbesserung der (digitalen) Gestaltung und der Rollenwandel bei den Kartennutzerinnen und -nutzern.

Und ich wünsche wieder viel Freude beim Lesen des neuen Heftes,

herzlichst,

Ihr

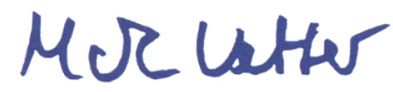

